Hugo Aimar, Department of Mathematics-IMAL, Facultad de Ingeniería Química, Universidad Nacional del Litoral and CONICET, Argentina.

e-mail: haimar@intec.unl.edu.ar

Liliana Forzani, Department of Mathematics-IMAL, Facultad de Ingeniería Química, Universidad Nacional del Litoral and CONICET, Argentina.

e-mail: forzani@math.umn.edu

\title{
ON THE BESICOVITCH PROPERTY FOR PARABOLIC BALLS
}

\begin{abstract}
Let $p \geq 1$ and $a_{1}, \ldots, a_{n}$ be positive given numbers. We prove that, the family of all solids of $\mathcal{R}^{n}$ of the type $\sum_{i=1}^{n}\left(\frac{\left|x_{i}\right|}{r^{a_{i}}}\right)^{p}<1, r>0$ satisfies the Besicovitch covering lemma if and only if $p \geq \frac{\max a_{i}}{\min a_{i}}$.
\end{abstract}

\section{Introduction}

Let $\mathcal{U}=\left\{U_{\alpha}: \alpha \in \mathcal{R}^{+}\right\}$be an increasing family of bounded and open neighborhoods of the origin in $\mathcal{R}^{n}$. Given a Borel measure $\mu$ on $\mathcal{R}^{n}$, a centered maximal Hardy-Littlewood operator associated to $\mu$ and $\mathcal{U}$ can be defined by

$$
M_{\mu, \mathcal{U}} f(x)=\sup _{\alpha \in \mathcal{R}^{+}} \frac{1}{\mu\left(x+U_{\alpha}\right)} \int_{x+U_{\alpha}}|f(y)| d \mu(y),
$$

for every Borel measurable function $f$. The boundedness of this operator on Lebesgue spaces with measure $\mu$ is a basic result for any further real analysis associated to the setting defined by $\mathcal{U}$ and $\mu$. It is well known that these boundedness properties are intimately related to the covering properties of the family $\mathcal{U}$ : they are usually proved using Wiener or Besicovitch type covering lemmas. Wiener type covering lemmas hold true with very mild conditions on $\mathcal{U}$, but for general non-doubling measures Besicovitch type covering lemmas are the basic useful tool.

Key Words: Besicovitch, covering lemma, real variable theory

Mathematical Reviews subject classification: Primary 42B99; Secondary 26 B99.

Received by the editors July 7, 1999 
Let us introduce the Besicovitch property for a general family $\mathcal{U}$. We shall say that the family $\mathcal{U}$ satisfies the Besicovitch property (BP) if there exists a constant $C$, depending only on $\mathcal{U}$ and $n$, such that for each family $\left\{x_{i}+\mathcal{U}_{\alpha_{i}}: i \in I\right\}$ of translated members of $\mathcal{U}$, such that $\left\{x_{i}: i \in I\right\}$ is a bounded set, there is a subset $J$ of $I$ such that

(CP) $\left\{x_{i}: i \in I\right\} \subset \bigcup_{j \in J}\left(x_{j}+\mathcal{U}_{\alpha_{j}}\right)$ (Covering Property);

and

(OP) $\sum_{j \in J} \chi_{\left\{x_{j}+\mathcal{U}_{\alpha_{j}}\right\}}(x) \leq C$ (Overlapping Property).

In this note we shall deal with the special case of balls associated to distances or quasi-distances given by non isotropic dilations on $\mathcal{R}^{n}$. Let $A$ be a diagonal real matrix with positive eigenvalues $a_{1} \leq a_{2} \leq \cdots \leq a_{n}$. Let $\tau_{\lambda}(x)=e^{A \log \lambda} x=\left(\lambda^{a_{1}} x_{1}, \cdots, \lambda^{a_{n}} x_{n}\right), \lambda>0$. Let $p \geq 1$. For $x \neq 0$, the continuous function

$$
\phi(\rho)=\left\{\sum_{i=1}^{n}\left(\frac{\left|x_{i}\right|}{\rho^{a_{i}}}\right)^{p}\right\}^{\frac{1}{p}}
$$

is strictly decreasing with $\lim _{\rho \rightarrow 0^{+}} \phi(\rho)=+\infty$ and $\lim _{\rho \rightarrow+\infty} \phi(\rho)=0$. Then, given $x \in \mathcal{R}^{n}-\{0\}$, the equation $\left\|\tau_{\frac{1}{\rho}}(x)\right\|_{p}=\phi(\rho)=1$ has only one solution which we call $\rho_{p}(x)$. One can check that the function $\rho_{p}$ defines a quasi-distance (which means $\rho_{p}(x, y) \leq K_{p}\left(\rho_{p}(x, z)+\rho_{p}(z, y)\right)$ (See [4])) on $\mathcal{R}^{n}$ with constant $K_{p}$. The $\rho_{p}$-balls are the solids of $\mathcal{R}^{n}$ given by

$$
B_{r}=\left\{\left(x_{1}, \ldots, x_{n}\right) \in \mathcal{R}^{n}:\left(\frac{\left|x_{1}\right|}{r^{a_{1}}}\right)^{p}+\ldots+\left(\frac{\left|x_{n}\right|}{r^{a_{n}}}\right)^{p}<1\right\}
$$

These parabolic balls associated to a non-doubling Gaussian measure appear for example in the real analysis setting associated with the generalized Ornstein-Uhlenbeck operators coming from the equation $\Delta u-2 A x . \nabla u=0$, where $A$ is a matrix in $\mathcal{R}^{n} \times \mathcal{R}^{n}$ (see [2]).

The main result in this note is that the $\mathbf{B P}$ for the family of $\rho_{p}$-balls depends on the condition number of the matrix $A$ given by the ratio $a_{n} / a_{1}$.

Theorem 1.1. Let $p \geq 1$. Let $\mathcal{U}$ be the family of all $B_{r}, r>0$ given by (3). Then, $\mathcal{U}$ satisfies $\mathbf{B P}$ if and only if $\frac{a_{n}}{a_{1}} \leq p \leq+\infty$.

\section{The only if part of the Theorem.}

Given $a_{1}, \ldots a_{n}$, and $p \geq 1$ we will say that $\mathcal{E}$ is an $\left(a_{1}, \ldots, a_{n} ; p\right)$-ellipsoid of radius $r>0$ if, for some coordinate system, its equation becomes 


$$
\sum_{i=1}^{n}\left(\frac{\left|x_{i}\right|}{r^{a_{i}}}\right)^{p}=1
$$

Certainly, $\mathcal{E}$ is the boundary of a $\rho_{p}$-ball.

Proposition 2.1. Let $0<a_{1} \leq a_{2} \leq \cdots \leq a_{n}$. Let $Q=\left(y_{1}, \ldots, y_{n}\right)$ be a point in $\mathcal{R}^{n}$. Let $\delta \in(0,1)$ be given. Let $P=\left(y_{1}+\delta, \ldots, y_{n-1}+\delta, y_{n}+y\right)$ with $y>0$ and $\delta^{p} \sum_{i=1}^{n-1} y^{-p a_{i} / a_{n}}<1$. Then, there exits $r>0$ such that the $\left(a_{1}, \ldots a_{n} ; p\right)$-ellipsoid of radius $r$ with axes parallel to the coordinate system and with one vertex at $P$ passes through $Q, r(\delta) \rightarrow+\infty$ as $\delta \rightarrow 0$ and if $P^{\prime}$ is the opposite vertex to $P, d(P, Q) \leq d\left(P^{\prime}, Q\right)$.

The proof of Proposition 2.1 is an easy consequence of the following lemma

Lemma 2.1. Let $y>0$ and $p \in\left[1, \frac{a_{n}}{a_{1}}\right)$, and $0<a_{1} \leq a_{2} \leq \cdots \leq a_{n}$. Then, for each $\delta>0$ small enough, $\left(\delta^{p} \sum_{i=1}^{n-1} y^{-p a_{i} / a_{n}}<1\right)$, the equation

$$
\delta^{p} \sum_{i=1}^{n-1} \frac{1}{r^{p a_{i}}}+\left|\frac{y-r^{a_{n}}}{r^{a_{n}}}\right|^{p}=1
$$

has at least one solution $r(\delta)$ greater than or equal to $y^{\frac{1}{a_{n}}}$, such that $r(\delta) \rightarrow$ $+\infty$ when $\delta \rightarrow 0$.

Proof. With $s=r^{-p a_{1}}$ our problem is equivalent to find the solution of $f(s)=1$ with $0<s<y^{-p a_{1} / a_{n}}$, where $f(s)=\delta^{p} \sum_{i=1}^{n-1} s^{a_{i} / a_{1}}+\left|s^{a_{n} / a_{1} p} y-1\right|^{p}$. Notice that $f(0)=1$ and $f^{\prime}(0)=\delta^{p}>0$ (If $a_{1}=\cdots=a_{k}<a_{k+1} \leq \ldots$, then $f^{\prime}(0)=k \delta^{p}>0$. Since $f\left(y^{-p a_{1} / a_{n}}\right)=\delta^{p} \sum_{i=1}^{n-1} y^{-p a_{i} / a_{n}}<1$ we must have a solution of $f(s)=1$ with $0<s<y^{-p a_{1} / a_{n}}$. Now since $r(\delta) \geq y^{1 / a_{n}}$, we have

$$
\begin{aligned}
y & =r^{a_{n}}\left[1-\left(1-\delta^{p} \sum_{i=1}^{n-1} \frac{1}{r^{p a_{i}}}\right)^{1 / p}\right] \\
& \simeq r^{a_{n}} \delta^{p} \sum_{i=1}^{n-1} \frac{1}{r^{p a_{i}}}
\end{aligned}
$$

from which, since $y$ is constant, we get the desired result.

Let $0<a_{1} \leq \cdots \leq a_{n}$ and $p \in\left[1, \frac{a_{n}}{a_{1}}\right)$ be given. Let $\rho=\rho_{p}$ be the quasi distance given by the numbers $a_{1}, \ldots, a_{n}$ and $p$. With the help of Proposition 2.1 we will construct a sequence of $\rho$-balls with unbounded overlapping such 
that if $x_{i}$ is the center of $B_{i}$, then $x_{i} \notin B_{j}$ for $j \neq i$. So that every ball is needed if we want to cover the centers.

Let $B_{o}=B(0,1)$. Let $Q_{1}=0$. Choose $y>0$ and $\delta_{1}>0$ so that $\delta_{1}^{p} \sum_{i=1}^{n-1} y^{-p a_{i} / a_{n}}<1$, and $P_{1}=\left(\delta_{1}, \ldots, \delta_{1}, y\right)$ belongs to the first $2^{n}$-quadrant of $B_{o}$. From Proposition 2.1 if we choose $\delta_{1}$ small enough, we can pick an $\left(a_{1}, \ldots, a_{n} ; p\right)$-ellipsoid, and then its interior $B_{1}$, in such a way that the center $Q_{2}$ of $B_{1}$ does not belong to $B_{o}$, neither the center of $B_{o}$ to $B_{1}$. Choose $P_{2}=\left(\delta_{1}+\delta_{2}, \ldots, \delta_{1}+\delta_{2}, z\right)$ so that $\delta_{2}<1$ is small enough and $\delta_{2}^{p} \sum_{i=1}^{n-1} z^{-p a_{i} / a_{n}}<1$. Now, because of Proposition 2.1 for $\delta_{2}$ small enough we can find an $\left(a_{1}, \ldots, a_{n} ; p\right)$-ellipsoid and then its interior $B_{2}$, in such a way that the center does not belong to $B_{o} \cap B_{1}$ and, clearly, the centers of $B_{o}$ and $B_{1}$ do not belong to $B_{2}$. Since $B_{o} \cap B_{1} \cap B_{2}$ in a non-empty open set of points with first $n-1$ coordinates larger than the first of $P_{2}$, the process can be iterated to obtain the desired result.

Notice that one can easily construct an atomic measure $\mu$ on the Borel sets of $\mathcal{R}^{2}$ in such a way that the centered maximal function defined with this family of balls is not of weak type $(1,1)$ with respect to this measure.

\section{The if part of the theorem.}

In the sequel $B(x, r)$ will denote the $\rho_{p}$-balls and $S(x, r)$ the $\|\cdot\|_{p}$-balls in $\mathcal{R}^{n}$ $\left(\|\cdot\|_{p}\right.$ is the usual $p$ norm on $\left.\mathcal{R}^{n}\right)$; of course for any $x, B(x, 1)=S(x, 1)$.

Theorem 3.1. Let $A$ be a fixed $n \times n$ diagonal matrix with positive eigenvalues $a_{1} \leq a_{2} \leq \cdots \leq a_{n}$ and $p \geq \frac{a_{n}}{a_{1}}$. Then the family of $\rho_{p}$-balls has the $\mathbf{B P}$.

Proposition 3.2. Let $x, z \in \mathcal{R}$, such that $x z \geq 0$. Then for all $\lambda \in[0,1]$ and for all $1 \leq q \leq p$ we have

$$
|\lambda z-x|^{p} \leq \lambda^{q}|x-z|^{p}+\left(1-\lambda^{q}\right)|x|^{p} .
$$

\section{Proof of TheOREM 3.1.}

Let $\left\{B\left(x_{\alpha}, r_{\alpha}\right): \alpha \in I\right\}$ be a family of $\rho_{p}$ balls whose centers are in a bounded set. Let $H=\sup \left\{r_{\alpha}: \alpha \in I\right\}$. Suppose that $H=+\infty$. Since for some $r>0$ we have $\left\{x_{\alpha}: \alpha \in I\right\} \subset B(0 ; r)$, choosing $\beta \in I$ such that $r_{\beta}>\rho_{p}$-diameter of $\left\{x_{\alpha}: \alpha \in I\right\}$, we get $\left\{x_{\alpha}: \alpha \in I\right\} \subset B\left(x_{\beta} ; r_{\beta}\right)$.

Suppose now that $H<+\infty$. Let $E^{1}=E_{1}^{1}=\left\{x_{\alpha}: \alpha \in I\right.$ and $\left.\frac{H}{2}<r_{\alpha} \leq H\right\}$. We take $x_{1} \in E_{1} ; B_{1}^{1}=B\left(x_{1} ; r_{1}\right)$. Assume that $E_{1}^{1} ; E_{2}^{1} ; \ldots ; E_{k-1}^{1} ; B_{1}^{1} ; B_{2}^{1} ; \ldots$; $B_{k-1}^{1}$ are defined, we take $E_{k}^{1}=E^{1}-\cup_{i=1}^{k-1} B_{i}^{1}$; and choose $x_{k} \in E_{k}^{1}$ and $B_{k}^{1}=B\left(x_{k} ; r_{k}\right)$. The process stops producing a finite sequence $\left\{B_{i}^{1}\right\}_{i=1}^{m_{1}}$ that 
cover $E_{1}$. In fact, from our selection $B\left(x_{i}^{1} ; \frac{H}{2^{2} K}\right) \cap B\left(x_{j}^{1} ; \frac{H}{2^{2} K}\right)=\emptyset$ for $i \neq j$ and they are subsets of $\left\{x: \rho_{p}\left(x,\left\{x_{\alpha}: \alpha \in I\right\}\right)<H\right\}$ which is bounded. Note that the number of balls $m_{1}$ depends only on $K, n, H$ and of the numbers $a_{1}, \ldots, a_{n},(K$ is the constant in the quasi-triangular inequality).

Now, assume that $E^{1} ; \ldots ; E^{l-1} ; B_{i}^{j}\left(j=1, \ldots, l-1 ; k=1, \ldots, m_{j}\right)$ are defined, we take $E^{l}=E_{1}^{l}=\left\{x_{\alpha}: \alpha \in I\right.$ and $\left.\frac{H}{2^{l}}<r_{\alpha} \leq \frac{H}{2^{l-1}}\right\}-\cup_{j=1}^{l-1} \cup_{i=1}^{m_{j}} B_{i}^{j}$; and we do the same that in the case $l=1$; we obtain then a finite sequence of balls covering $E^{l}$. Then, the sequence $\left\{B_{i}^{j}\right\}_{j, i}$ verify the $\mathbf{C P}$ property.

Let us now prove the OP. We claim that it is enough to prove

(i) $\sum_{i=1}^{m_{j}} \chi_{B_{i}^{j}}(z) \leq C_{1}$, for every $j$ and

(ii) $\sum_{j=1}^{+\infty} \chi_{\cup_{i=1}^{m_{j}} B_{i_{j}}^{j}}(z) \leq C_{2}$.

Indeed, assuming $(i)$ and $(i i)$, we have

$$
\sum_{j=1}^{\infty} \sum_{i=1}^{m_{j}} \chi_{B_{i}^{j}}(z) \leq \sum_{\left\{j: \exists i z \in B_{i}^{j}\right\}} \sum_{\left\{i: z \in B_{i}^{j}\right\}} \chi_{B_{i}^{j}}(z) \leq C_{1} C_{2} .
$$

(i) Let $z$ be a point in $M$ of the balls $\left\{B_{i}^{j}\right\}_{i=1}^{m_{j}}, B_{i_{1}}^{j}, \ldots, B_{i_{M}}^{j}$. As in the case $j=1$ we have $\left\{B\left(x_{i_{s}}^{j}, \frac{H}{K 2^{j+1}}\right)\right\}_{s=1}^{M}$ disjoint and $B\left(x_{i_{s}}^{j} ; \frac{H}{K 2^{j+1}}\right) \subset B\left(z ; \frac{H}{2^{j}}\right)$. Then, since the Lebesgue measure of a $\rho$-ball of radius $r$ is equivalent to $r^{\text {trace } A}$, we easily see that $M$ is an absolute constant.

(ii) Let $z$ be a point in $M$ of the balls $\left\{B_{i_{j}}^{j}:=B_{j}\right\}_{j}$. Since $\rho_{p}$ is translation invariant, we may suppose that $z=0$. For simplicity of notation let us assume that $0 \in \cap_{j=1}^{M} B_{j}$, with $B_{j}$ preceding $B_{j+1}$ in the selection procedure. Since $x_{k} \notin \cup_{j=1}^{k-1} B_{j}$, we get $\tau_{\frac{1}{r_{k}}}\left(x_{k}\right) \notin \cup_{j=1}^{k-1} \tau_{\frac{1}{r_{k}}}\left(B_{j}\right)$. In the case in which $A$ is a constant times the identity matrix, $\tau_{t}(z)$ are straight lines so that the inclusion $\tau_{\frac{1}{r_{j}}}\left(B_{j}\right) \subset \tau_{\frac{1}{r_{k}}}\left(B_{j}\right)$ for $r_{k} \leq r_{j}$, is clear. For general $A$ we will prove later that

$$
\tau_{\frac{1}{r_{j}}}\left(B_{j}\right) \cap Q_{j} \subset \tau_{\frac{1}{r_{k}}}\left(B_{j}\right) \quad \text { for } j<k
$$

where $Q_{j}$ is the quadrant of $\mathcal{R}^{n}$ such that $x_{j} \in Q_{j}$.

First assume that (5) holds. Then clearly

$$
\tau_{\frac{1}{r_{k}}}\left(x_{k}\right) \notin \bigcup_{j=1}^{k-1}\left(\tau_{\frac{1}{r_{j}}}\left(B_{j}\right) \cap Q_{j}\right) .
$$

Now, (6) allows us to finish the proof of the theorem, since we can find $\xi_{j} \in \tau_{\frac{1}{r_{j}}}\left(B_{j}\right) \cap Q_{j}$ for $j=1, \ldots, M$ such that for $\epsilon=\left(4 n^{\frac{1}{p}}\right)^{-1}$ we have that $\left\{S\left(\xi_{j}, \frac{\epsilon}{2}\right)\right\}_{j=1}^{M}$ are pairwise disjoint, and $\left\|\xi_{j}\right\|_{p} \leq \frac{5}{4}$. 
Indeed, since $\tau_{\frac{1}{r_{j}}}\left(B_{j}\right)=S\left(\tau_{\frac{1}{r_{j}}} x_{j}, 1\right)$, we can pick $\xi_{j}$ with coordinates equal to those of $\tau_{\frac{1}{r_{j}}}\left(x_{j}\right)$ plus or minus $\epsilon$ such that $S\left(\xi_{j}, \epsilon / 2\right) \subset \tau_{\frac{1}{r_{j}}}\left(B_{j}\right) \cap Q_{j}$ and $\left\|\xi_{j}-\tau_{\frac{1}{r_{j}}}\left(x_{j}\right)\right\|_{p} \leq \frac{1}{4}$. Suppose that there exists $w \in S\left(\xi_{j}, \frac{\epsilon}{2}\right) \cap S\left(\xi_{k}, \frac{\epsilon}{2}\right)$ with $j<k$. Then, $\tau_{\frac{1}{r_{j}}}\left(x_{j}\right)$ and $\tau_{\frac{1}{r_{k}}}\left(x_{k}\right)$ belongs to the same quadrant, so that $\tau_{\frac{1}{r_{k}}}\left(x_{k}\right) \in Q_{j}$. On the other hand

$$
\begin{aligned}
\left\|\tau_{\frac{1}{r_{k}}}\left(x_{k}\right)-\tau_{\frac{1}{r_{j}}}\left(x_{j}\right)\right\|_{p} & \leq\left\|\tau_{\frac{1}{r_{k}}}\left(x_{k}\right)-\xi_{k}\right\|_{p}+\left\|\xi_{k}-\xi_{j}\right\|_{p}+\left\|\tau_{\frac{1}{r_{j}}}\left(x_{j}\right)-\xi_{j}\right\|_{p} \\
& \leq \frac{3}{4}
\end{aligned}
$$

which is a contradiction since $\tau_{\frac{1}{r_{k}}}\left(x_{k}\right) \notin \tau_{\frac{1}{r_{j}}}\left(B_{j}\right) \cap Q_{j}$.

Let us prove (5). To show that $z \in \tau_{\frac{1}{r_{k}}}\left(B_{j}\right)$ whenever $z \in \tau_{\frac{1}{r_{j}}}\left(B_{j}\right) \cap Q_{j}$ is the same as proving that $\tau_{\frac{r_{k}}{r_{j}}}(z) \in \tau_{\frac{1}{r_{j}}}\left(B_{j}\right)=B\left(\tau_{\frac{1}{r_{j}}}\left(x_{j}\right), 1\right)$. As $1 \leq \frac{a_{n}}{a_{s}} \leq \frac{a_{n}}{a_{1}}$, and $z \in Q_{j}$ using Proposition 3.2 with $\lambda=\left(\frac{r_{k}}{r_{j}}\right)^{a_{s}}, q=\frac{a_{n}}{a_{s}}, x=\left(\frac{1}{r_{j}}\right)^{a_{s}} x_{j}^{(s)}$, $z=z^{(s)}$ with $s=1, \ldots, n$, where $y^{(s)}$ is the $s$ - th coordinate of $y$, we have for $p \geq \frac{a_{n}}{a_{1}}$ that

$$
\begin{aligned}
& \left\|\tau_{\frac{r_{k}}{r_{j}}}(z)-\tau_{\frac{1}{r_{j}}}\left(x_{j}\right)\right\|_{p}^{p}=\sum_{s=1}^{n}\left|\left(\frac{r_{k}}{r_{j}}\right)^{a_{s}} z^{(s)}-\left(\frac{1}{r_{j}}\right)^{a_{s}} x_{j}^{(s)}\right|^{p} \\
\leq & \sum_{s=1}^{n}\left(\left(\frac{r_{k}}{r_{j}}\right)^{a_{n}}\left|\frac{x_{j}^{(s)}}{r_{j}^{a_{s}}}-z^{(s)}\right|^{p}+\left(1-\left(\frac{r_{k}}{r_{j}}\right)^{a_{n}}\right)\left|\frac{x_{j}^{(s)}}{r_{j}^{a_{s}}}\right|^{p}\right) \\
= & \left(\frac{r_{k}}{r_{j}}\right)^{a_{n}} \sum_{s=1}^{n}\left|\frac{x_{j}^{(s)}}{r_{j}^{a_{s}}}-z^{(s)}\right|^{p}+\left(1-\left(\frac{r_{k}}{r_{j}}\right)^{a_{n}}\right) \sum_{s=1}^{n}\left|\frac{x_{j}^{(s)}}{r_{j}^{a_{s}}}\right|^{p} ;
\end{aligned}
$$

now, since $z \in \tau_{\frac{1}{r_{j}}}\left(B_{j}\right)$ and $0 \in \tau_{\frac{1}{r_{j}}}\left(B_{j}\right)$ the last term is bounded by one.

Proof of Proposition 3.2. The desired inequality for $q=1$ follows from the convexity of $|x|^{p}$. To prove (4) for $q=p$ let us note that dividing (4) by $|x|^{p}$ and taking $h=\frac{|z|}{|x|}=\frac{z}{x} \geq 0$, (4) for $q=p$ is equivalent to

$$
|\lambda h-1|^{p} \leq \lambda^{p}|h-1|^{p}+\left(1-\lambda^{p}\right) .
$$

Taking $v=\lambda h$, this is equivalent to

$$
|v-1|^{p} \leq|\lambda-v|^{p}+\left(1-\lambda^{p}\right) \text { for all } v \geq 0 .
$$


Let $f$ be the function $f(v)=|v-1|^{p}-|\lambda-v|^{p}-\left(1-\lambda^{p}\right)$. Since $\lambda \leq 1$, we have that $f(v) \leq 0$ for $v \geq 1$. On the other hand, since $f(0)=0$ and $f^{\prime}(v)=-p|1-v|^{p-1} \operatorname{sign}(1-v)-p|v-\lambda|^{p-1} \operatorname{sign}(v-\lambda) \leq 0$ for $v \leq 1$ we have the inequality for all $v \geq 0$.

Now, let us prove (4) for a general $q, 1 \leq q \leq p$. Since (4) is true for $q=1$ and $q=p$, then for all $0 \leq \alpha \leq 1$ we have that

$$
\begin{aligned}
|\lambda z-x|^{p} \leq & \alpha\left(\lambda|x-z|^{p}+(1-\lambda)|x|^{p}\right) \\
& \quad+(1-\alpha)\left(\lambda^{p}|x-z|^{p}+\left(1-\lambda^{p}\right)|x|^{p}\right) \\
= & \left((1-\alpha) \lambda^{p}+\alpha \lambda\right)|x-z|^{p}+\left((1-\lambda) \alpha+\left(1-\lambda^{p}\right)(1-\alpha)\right)|x|^{p} .
\end{aligned}
$$

Now, since for each $0 \leq \lambda \leq 1$ there is an $\alpha$ such that $(1-\alpha) \lambda^{p}+\alpha \lambda=\lambda^{q}$, we get (4) for general $q$.

Let us observe that, since after an orthogonal transformation every diagonalizable matrix $A$ with positive eigenvalues can be written in diagonal form, if $A$ has positive eigenvalues $a_{1} \leq a_{2} \leq \cdots \leq a_{n}$ with $\frac{a_{n}}{a_{1}} \leq 2$, then the balls associated to the distance induced by $A$ with $p=2$ have the $\mathbf{B P}$.

Let us finally notice that when $p=\infty$ the balls become one parameter families of rectangles that satisfy the $\mathbf{B P}$ for every size of the condition number of $A$.

We would like to point out that the dyadic argument to reduce the infinite to the finite case used as the starting point of the proof is due to A. P. Calderón ([1]) and that related results on the Heisenberg group can be found in [3].

\section{References}

[1] A. Calderón, Oral communication. Universidad de Buenos Aires, 1982.

[2] M. Fuhrman, Analyticity of transition semigroups and closability of bilinear forms in Hilbert spaces. Studia Math. 115(1) (1995).

[3] E. Sawyer and R. Wheeden, Weighted inequalities for fractional integrals on Euclidean and homogeneous space, Amer. J. Math. 114(2), (1992), 813-874.

[4] E.Stein, and S. Wainger, Problems in harmonic analysis related to curvature. Bull. Amer. Math. Soc. 84(6) (1978), 1239-1295. 
